\title{
Performances of volume phase holographic grating for space applications: study of the radiation effect
}

\author{
Jérôme Loicq, ${ }^{1, \star}$ Luis Miguel Venancio, ${ }^{2}$ Yvan Stockman, ${ }^{1}$ and Marc P. Georges ${ }^{1}$ \\ ${ }^{1}$ Centre spatial de Liège-University of Liège, Liège Science Park, Avenue du Pré Aily, \\ 4031 Angleur/Liege, Belgium \\ 2European Space Agency ESTEC, Keplerlaan 1, 2200 AG Noordwijk ZH, The Netherlands \\ *Corresponding author: J.Loicq@ulg.ac.be
}

Received 21 August 2013; revised 14 October 2013; accepted 14 October 2013; posted 23 October 2013 (Doc. ID 196141); published 25 November 2013

\begin{abstract}
The special properties of volume phase holographic gratings make them promising candidates for spectrometry applications where high spectral resolution, low levels of straylight, and low polarization sensitivity are required. Therefore it is of interest to assess the maturity and suitability of volume phase holographic gratings as enabling technologies for future space missions, with demanding requirements for spectrometry. One of the main areas of research is related to grating ageing under space radiation. In the present paper, two volume grating technologies are analyzed and compared under gamma irradiation. The performances of both technologies, the photo-thermo-refractive glass and the Dichromated Gelatin, are tested on samples and assessed in the $H_{\alpha}$ and near-infrared bands. The diffraction efficiency degradation under gamma irradiation is assessed. (C) 2013 Optical Society of America

OCIS codes: (050.7330) Volume gratings; (050.1970) Diffractive optics; (120.6085) Space instrumentation; (350.5610) Radiation.

http://dx.doi.org/10.1364/AO.52.008338
\end{abstract}

\section{Introduction}

Holography is now a well-established technique and has found many applications. An example of an innovative application is the volume phase holographic (VPH) grating [1,2]. The special properties of VPH gratings make them interesting for spectrometry applications, where high spectral resolution, low levels of straylight, and low polarization sensitivity are required $[\underline{3}, \underline{4}]$. VPH gratings are produced by illuminating a photosensitive substrate with overlapping coherent light beams. The resulting interference pattern is then permanently recorded in the volume of the substrate as a periodic variation of

$1559-128 \mathrm{X} / 13 / 348338-09 \$ 15.00 / 0$

(C) 2013 Optical Society of America the refractive index of the substrate material. Once recorded, these gratings spatially disperse the spectrum of an incident light beam, like other dispersive elements, such as prisms and conventional gratings. In most optical designs for space and astronomy applications, surface relief (SR) gratings are used $[5,6]$. In comparison with SR gratings, the VPH gratings have interesting advantages. The main advantages are higher peak diffraction efficiency, lower polarization dependency, weaker level of scattered light, and higher spectral and spatial selectivity. Such gratings are presently studied with the objective to implement them in future space missions. The case of Earth observation missions is under consideration in this paper. Specifically, such gratings are foreseen for use in hyper-spectral imaging systems. Earth observation missions require more 
efficient technologies to develop new instruments concept to analyze, with increased accuracy, phenomena related to the oceans, the land, vegetation, icecap evolution, climate, etc.

To ensure an Earth observation mission viability and success, the VPH grating components must withstand the harsh environment of space with little or no degradation of performance. In particular, these gratings have to resist the thermal vacuum environment, radiation (electron, proton, gamma, heavy ions), atomic oxygen, mechanical vibrations, etc. In this paper, we focused on an analysis of VPH diffraction grating efficiency behavior under gamma radiation.

The optical and functional performances required for space missions are essentially focused on wavefront quality, polarization sensitivity, and diffraction efficiency. Two VPH candidate technologies were analyzed and compared: The first technology involves a dichromated gelatin (DCG) that is made with a thin film (tens of micrometers thick) of photosensitive DCG, sandwiched between two glass plates. This type of VPH is already widely used in ground-based astronomy applications $[3,4]$. The second technology is photo-thermo-refractive (PTR) glass. The grating is recorded in the bulk of the glass substrate. This technology allows the manufacturing of thicker (several millimeters thick) VPH gratings, which produces a narrower spectrum of the diffracted beam $[7,8]$. The main selection criteria for these two technologies are performance, in terms of diffraction efficiency, and commercial availability as a bulky component without the need of further processing.

For the present analysis, two bands have been selected: $H_{\alpha}(646-666 \mathrm{~nm})$ and near-infrared (NIR) (750-775 nm) following European Space Agency (ESA) requirements for Earth observation missions. Extrapolation to other visible and infrared bands can be made easily. The performances of these gratings were experimentally assessed under gamma radiation.

In this paper, the main properties of VPH gratings will be described, and, specifically, the characteristics of gratings realized on DCG and photothermal refractive glass. Next, the gratings will be characterized in terms of diffraction efficiency, polarization sensitivity, super-blazed properties, and out-of bands characteristics. Finally, the effect of gamma radiation on these parameters will be assessed.

\section{A. Volume Phase Holographic Grating}

A VPH grating is a periodic phase structure (refractive index spatial modulation $\Delta n$ ) written in the bulk of a layer of photosensitive material. The interference pattern used for the definition of the VPH can be oriented arbitrarily with respect to the faces of the substrate used. Such phase holograms can work in transmission or reflection modes. Figure $\underline{1}$ presents those two configurations. In the first case, refractive index modulation is mostly perpendicular

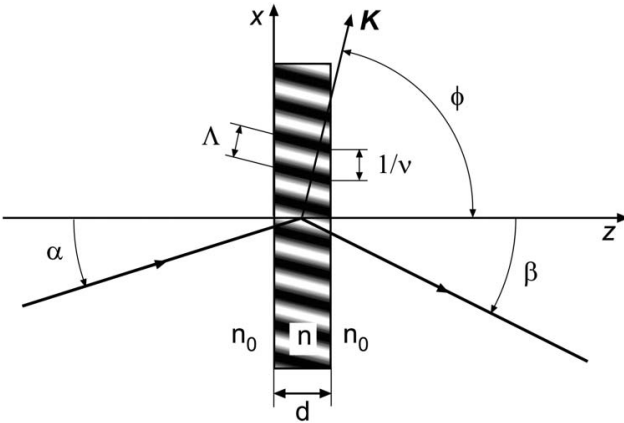

(a)

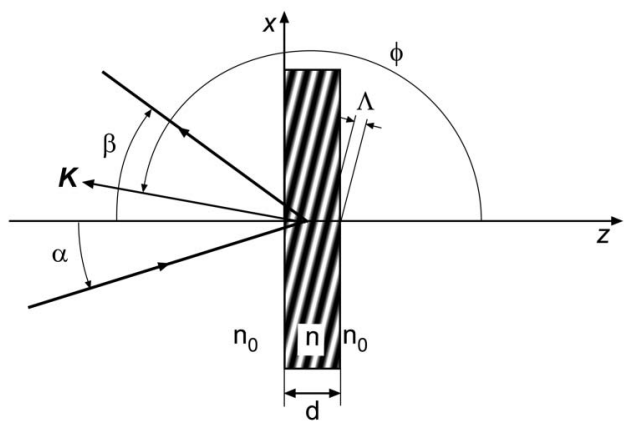

(b)

Fig. 1. Types of slanted phase holograms (a) in transmission mode and (b) in reflection mode. $\alpha$ and $\beta$ are considered inside the holographic material.

to the photosensitive substrate and, in the second case, the refractive index modulation is close to parallel to the substrate. Early in the genesis of holography developments, these specific properties were pointed out and modeled by Kogelnik [9].

In transmission mode, depending on the thickness of the VPH grating substrate, the diffraction regime can be either the Raman-Nath regime, i.e., several diffraction orders are present, or the Bragg regime, where only one diffraction order is present. In the latter case, the VPH gratings are often called volume Bragg gratings (VBG). This occurs when some ratios between grating parameters (thickness, line frequency, refractive index modulation) are reached and, therefore, the diffraction behavior (efficiency versus wavelength, or incident angle) can be predicted using the mathematical model, coupled wave theory, developed by Kogelnik [9]. Otherwise, such results require the use of a well-established, high performing, and reliable numerical model known as rigorous-coupled wave analysis (RCWA) that was derived by Moharam and Gaylord [10,11].

Such VPHs are increasingly used in astronomy applications $[\underline{3}, \underline{4}]$ and are commercially available from a few companies worldwide. Different materials are used to realize VPH gratings [12]; for example, silver halides [13], DCG [4,14,15], photopolymers [16-18], and photochromic materials [19,20]. VPH gratings made on DCG are commonly used in ground-based astronomy applications. These gratings are made of a thin (tens of micrometers in thickness) film of photosensitive dichromate gelatin (DCG), sandwiched 
between two pieces of glass. Presently. a new photosensitive glass, the sodium-zinc aluminum-silicate glass doped with silver, cerium, and fluorine (also known as PTR glass, allows the manufacture of thicker (several millimeters) gratings, which produce a narrower spectrum of the diffracted beam [8]. PTR glass has demonstrated properties that would make VPH gratings an interesting alternative to bulk dispersive devices in the visible and NIR optical spectral range. In this paper, we focused our experimental analysis on VPH gratings made with DCG and PTR. These technologies have been selected by ESA first due to their achievable diffraction efficiency and, second, for their commercial availability as bulky components (like glass pieces) that would require no postprocessing.

\section{B. Dichromated Gelatin Grating}

The fabrication of DCG VPH gratings comprises the following steps. A thin film of photosensitized gelatin is deposited onto a glass (usually BK7) or fused silica substrate. The coated substrate is exposed to a holographic fringe pattern formed by the interference of two beams of coherent light from a common laser source. The exposed gelatin coating is processed in a series of liquid baths to achieve the desired level of refractive index modulation. Once the desired grating parameters have been achieved, a second plate of glass, or fused silica, is laminated over the processed grating film to protect it from the environment, using an optical adhesive, as shown in Fig. 2. Antireflection coatings can be applied to the substrates to reduce reflection losses.

The resulting physical structure is rugged, cleanable, stable, and has excellent optical characteristics. Note that the aperture of the gelatin grating film is usually smaller than that of the glass substrate and cover plate. The outer border is filled with optical adhesive that protects the edge of the gelatin film from moisture.

DCG has an average refractive index $n$ of about 1.5 and can be processed to deliver index modulations $\Delta n$ ranging from about 0.02 to about 0.10 in layers up to $20 \mu \mathrm{m}$ thick. Line densities of $300-6000$ $(\mathrm{ln} / \mathrm{mm})$ can be recorded in the material. In general, the index modulation produced in DCG is assumed to be sinusoidal. In []ㅡㄹ it is seen that DCG may have a useful transition window from $300 \mathrm{~nm}$ to $2.8 \mu \mathrm{m}$.

\section{Optical adhesive}

\begin{tabular}{|l|l|}
\hline Glass cover & \\
\hline Glass substrate & \\
\hline Gl & \\
\hline
\end{tabular}

Gelatin Grating

Fig. 2. Structure of a DCG-VPH transmission grating.
Note that there are strong constraints on the purity level of the DCG to minimize the impact of absorption bands that otherwise reside between 1 and $3 \mu \mathrm{m}$.

\section{Photo-Thermo-Refractive Gratings}

Concurrently to the DCG technology, VPH gratings can be fabricated by inducing a permanent refractive index change in a special PTR multicomponent silicate glass (BragGrate) after exposure to UV radiation followed by thermal treatment [7,8]. This allows fabrication of VPH gratings with absolute diffraction efficiency exceeding 99\%, thermal stability at up to $400^{\circ} \mathrm{C}$, a laser damage threshold of $40 \mathrm{~J} / \mathrm{cm}^{2}$ for $8 \mathrm{~ns}$ pulses, and a tolerance to $\mathrm{CW}$ laser radiation in the NIR region at least up to several tens of kilowatts per square centimeter. Such VPH gratings provide narrow spectral selectivity down to $20 \mathrm{pm}$ and narrow angular selectivity down to $100 \mu \mathrm{rad}$. These elements have low losses and can be used for a variety of applications in optoelectronics, analytical, and in the defense industries. It is seen that PTR gratings are substantially thicker than DCG gratings, and that the index modulation is much smaller. Their smaller bandwidth makes them more appropriate for filtering than for spectroscopy applications.

\section{Physical Parameters and Grating Characterization}

The use of a VPH grating in an optical design requires the understanding and the knowledge of its properties, among which the most important ones are diffraction efficiency, wave front error (WFE), polarization sensitivity, and the straylight generated by the device. The use of such components for space applications necessitates beforehand its qualification to the space environment. Space qualification of a new device involves very specific tests, during which device performances must be preserved. For example, the level of straylight generated by devices has to remain stable in time and the diffraction efficiency has to stay within an acceptable range of values [21]. Only at the end of the qualification process can the components, device or material be ready for use in space. The present study evaluates such a device and its survivability under gamma radiation. We define hereafter the main properties that need to be measured as functions of the gamma radiation level. For each of them, reference measurements have been performed prior to gamma irradiation.

Table 1 summarizes the characteristics of the VPH grating devices tested.

\section{A. Diffraction Efficiency}

The diffraction efficiency $\eta$ of a VPH grating could be roughly approached by the Kogelnik theory [9]. For a more accurate prediction, RCWA $[10,11]$ is mandatory. However, the approach of Kogelnik is enough to predict the effect of the first useful parameters such as grating thickness, index modulation, and blaze angle. Kogelnik's theory predicts that $\eta$ is a 
Table 1. Summary of the Characteristics of the VPH Grating Devices Tested

\begin{tabular}{lcc}
\hline & DCG & PTR \\
\hline Cl. ap. $^{a}\left(\mathrm{~mm}^{2}\right)$ & $100 \times 100$ & $50 \times 50$ \\
Central WL $^{b}(\mathrm{~nm})$ & 763 & 656 \\
BW-FWHM $^{c}(\mathrm{~nm})$ & 150 & 55 \\
Sp. freq. $^{d}(\mathrm{ln} / \mathrm{mm})$ & 1200 & 150 \\
Active layer $(\mathrm{mm})$ & 0.01 & 3 \\
Grating type & TRANS & TRANS \\
Slant angle & unslant & Unslant \\
Provider & Kaiser optical system, & Optigrate \\
& Inc. & Corp. \\
\hline
\end{tabular}

${ }^{a}$ Clear aperture.

${ }^{b}$ Central wavelength, corresponds to the design wavelength of the grating.

${ }^{c}$ Spectral bandwidth.

${ }^{d}$ Spatial frequency.

periodic function of the product of the index modulation by the thickness and divided by a term related to the grating frequency. For a given situation, there is an optimal value of the parameters combination. For example, with a given central wavelength and grating frequency, the optimal situation is obtained for a fixed value of the index modulation and thickness product. Consequently, it is possible to modulate the bandwidth value by increasing the refractive index modulation, while decreasing the grating layer thickness (within the limits of thick hologram regime and manufacturing possibility). The efficiency is certainly the most important parameter in the optical design. In fact, diffraction efficiency will determine the amount of light reaching the focal plane of the optical instrument. The higher the diffraction efficiency, the higher the light level on the detector. Consequently, from a radiometric point of view, the entrance pupil of the instrument could possibly be reduced until the imaging diffraction limit. Reducing the pupil means reducing the size of the payload and then the mass budget.

\section{Diffraction Efficiency Definition}

The diffraction efficiency definitions used in the present paper are:

- Absolute diffraction efficiency of the $n$th order:

$$
\frac{I^{n}}{I_{0}}=\eta_{\text {diff }}^{n} T_{1} T_{2},
$$

where $I^{n}$ is the transmitted light through the grating in the $n$th order, $I_{0}$ the incident light, $T_{1}$ and $T_{2}$ are the transmission coefficients of the entrance and exit interfaces of the grating, respectively.

- Relative diffraction efficiency of the $n$th order:

$$
\eta_{\mathrm{ref}}^{n}=\frac{I^{n}}{\sum_{i=0}^{\infty} I^{i}}
$$

From RCWA and Kogelnik theory, a grating is optimized for a central wavelength and a specific bandwidth. In that configuration, an index modulation $\Delta n$ and a VPH grating thickness are defined. The gratings must verify the Bragg condition:

$$
2 \Lambda \cos (\phi-\alpha)=\frac{\lambda}{n}
$$

where is the grating period, $\phi$ is the slant angle, $\alpha$ is the incident angle of the light considered inside the holographic medium, $\lambda$ the central wavelength, and $n$ the index of refraction of the holographic medium. By changing the incident angle $\alpha$, the Bragg condition is met for another central wavelength. Consequently, the usable spectral band could be tuned with the incidence angle and cover a very large spectral band. This property is called "super blazed" behavior.

\section{Diffraction Efficiency Characterization}

The gratings are characterized with a specific bench consisting of a fiber white collimated polarized light source (TE and TM). Transmitted or diffracted light is collected with a collimator assembly and injected into a fiber that directs the light to a monochromator detector. The grating is mounted on a rotation stage, while the detector collimator assembly is mounted on a goniometer. Each grating is measured with the following sequence: the dark measurement corresponds in dark conditions to the measurement of the detector noise; the reference measurement corresponds to the spectrum of the source lamp; and, finally, the performance measurement itself, which corresponds to the spectrum of the specimen of interest corrected from the dark and reference measurements. Diffraction efficiencies are also corrected from all-over components involved in the grating (cover, glue, substrate). Diffraction efficiency is then presented as an intrinsic property.

The diffraction efficiency of the VPH grating made of DCG is presented in Fig. 3 for both polarizations. The central curve on each subgraph is relative to the wavelength design of the grating: $763 \mathrm{~nm}$. The other curves correspond to a change of the angle of incidence of the entrance beam. As predicted by the theories of the volume grating and the Bragg Eq. (3), the central wavelength is dependent on the incident angle. "Super blazed" is materialized in Fig. 3 by the line connecting the maximum of each diffraction efficiency curve. The shape of the super blaze efficiency on TE and TM is different. For the wavelength design $(763 \mathrm{~nm})$ the measured efficiency is $97.8 \%$ at TE and $91.9 \%$ at TM.

The experimental diffraction efficiencies of the PTR grating are presented in Fig. 4. Both polarization properties are also presented on the graphs. As a DCG grating, PTR exhibits "super blazed" behavior. For its wavelength design, PTR efficiency is $48.6 \%$ at TE and $49.7 \%$ at TM. These efficiencies 


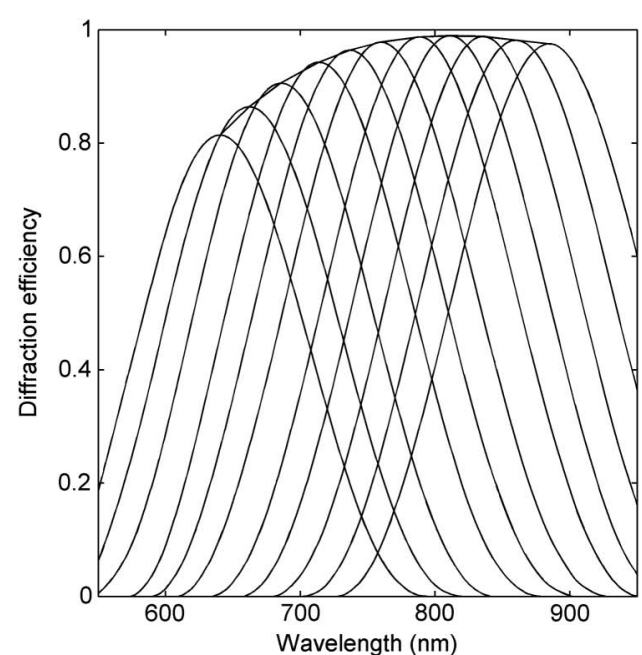

(a) TE polarisation

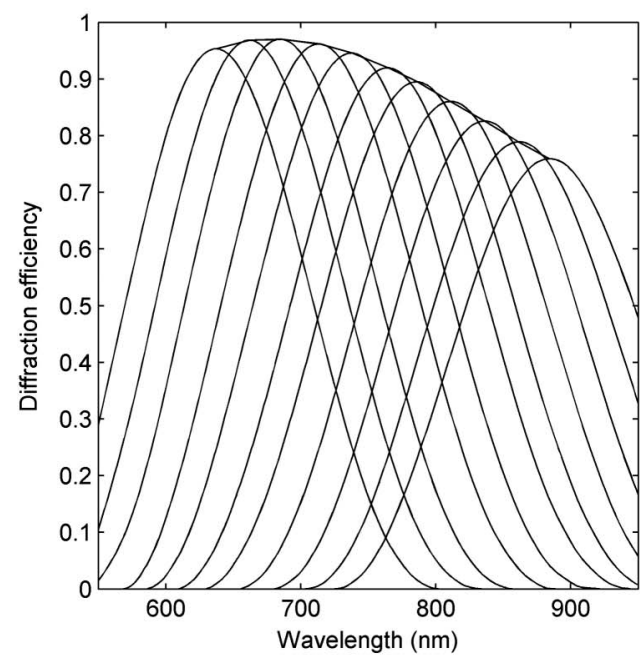

(b) TM polarisation

Fig. 3. Spectral characterization of the DCG grating designed for the NIR band. The curves represent diffraction efficiency acquired by (a) TE incident polarization and (b) TM incident polarization. Each curve corresponds to a given angle of incidence. The design wavelength is $763 \mathrm{~nm}$.

are clearly lower than the ones presented for the DCG grating. A rough analysis could conclude that PTR is less efficient than DCG; however, one cannot conclude so easily. From Kogelnik's Eq. [9], it is possible to derive the values of the bandwidth and the diffraction efficiency. These parameters are directly linked to the product $\Delta n \cdot d$, where $\Delta n$ is the index modulation recorded into the photosensitive material and $d$ its thickness. Moreover, the bandwidth of a VPH grating is inversely proportional to $d$ and directly proportional to $\Delta n$. PTR glasses are then disadvantaged for large bandwidths because of the available thicknesses for such material, which are in the order of magnitude of millimeters, when DCGs are in the range of few micrometers. Consequently, PTRs are more suitable for very thin bandwidths. In that case, high efficiencies of around $100 \%$ could

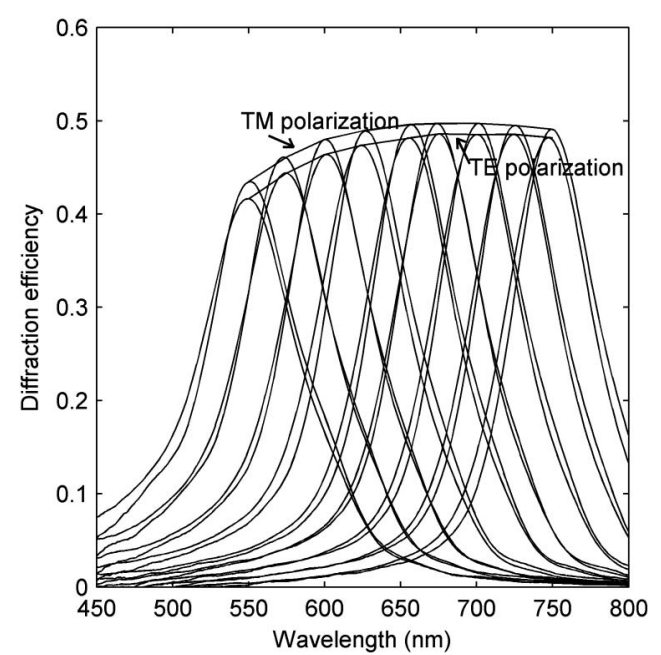

Fig. 4. Spectral characterization of the PTR grating designed for the $H_{\alpha}$ band: As presented in Fig. 3, PTR also exhibits super blazed properties. Each curve corresponds to a given angle of incidence. The design wavelength is $656 \mathrm{~nm}$.

be achieved as demonstrated in [7] and [8]. For large bandwidth and high efficiency, DCG gratings are more appropriate.

\section{B. Polarization Sensitivity}

Polarization sensitivity is a key issue on the spectrometric Earth Observation Mission. The instruments involved in the payload are generally sensitive to the polarization of the Earth's reflected radiance [22,23]. Moreover, most of those are not able to measure the incident polarization of the reflected light. In general, every reflective surface can modify incident light polarization. However, in Earth observation instruments, the most sensitive optical element to polarization is the grating [24]. Indeed, usually the grating is a SR grating working in a reflective mode. Such a grating is very sensitive to polarization and presents different diffractive performances for both incident polarizations, as demonstrated in many studies [25-27]. Consequently, the interpretation of the radiance measured by such an instrument could be impacted by significant errors. For example, observing an ocean depends largely on properties of reflection (specular and diffuse) of the sunlight on the water. In particular, polarization orientations induce Fresnel reflection. Consequently, the observation over a large swath on ground (or, equivalently, field-of-view) can give different results on the same scene depending on the satellite orientation. An instrument like Ocean Land Colour Instrument (OLCI) implemented in the Sentinel 3 ESA-mission [28] tackles this problem with the use of a scrambling window into the light path. This window induces a broadband circular polarization of the beam entering the diffraction grating.

The use of a VPH grating in such a hyperspectral instrument can drastically reduce the polarization sensitivity. 
Polarization sensitivity is defined as:

$$
S=\frac{\left|\eta_{\mathrm{TE}}-\eta_{\mathrm{TM}}\right|}{\left|\eta_{\mathrm{TE}}+\eta_{\mathrm{TM}}\right|}
$$

The polarization sensitivity is determined from the measurements of the diffraction efficiencies in both TE and TM polarization that are reported in Figs. 3(b) and 4 . Figure 5 shows the polarization sensitivity related to the $\mathrm{D} \overline{\mathrm{C}} \mathrm{G}$ grating under its spectral bands. The complete values of the polarization sensitivity are presented in Fig. 10, combined with the $\gamma$-radiation results.

\section{Out-of-Field Properties}

The use of VPH gratings is mainly foreseen in spectral imaging applications. Figure 6(a) represents the generic configuration of a very wide field "pushbroom" hyperspectral imager. In that case, the information related to the geometric aspects of the picture is in the perpendicular plane of the spectral information. Consequently, one of the interesting topics to analyze is the evolution of the diffraction efficiency with incidence in the perpendicular direction of the diffraction plane. This configuration is presented in Fig. 6(b). To compare the measurements, the Bragg condition has to always be kept valid.

Incidence $\delta_{\text {ext }}$ angles (often referred to as conical incidence) have been scanned from $0^{\circ}$ to $30^{\circ}$. Figure 7 presents the diffraction efficiency, respectively, for TE and TM polarizations. The diffraction efficiency is not largely impacted by the $\delta_{\text {ext }}$ angle. For the design wavelength this means a diffraction efficiency modification of about $2 \%-3 \%$, depending on the incident polarization. This dependence is observable on the two types of VPH gratings (PTR and DCG). However, the modest change in performance clearly

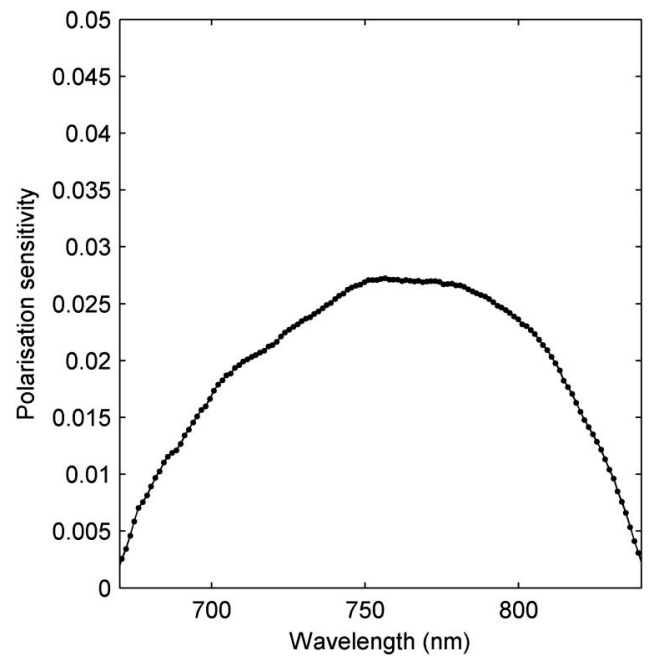

Fig. 5. Dependency of polarization sensitivity of DCG-NIR grating measured at a fixed incident angle on its spectral band peaked at $765 \mathrm{~nm}$.

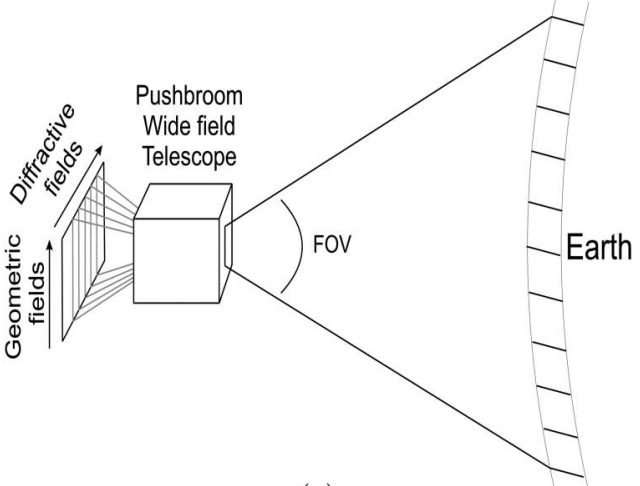

(a)

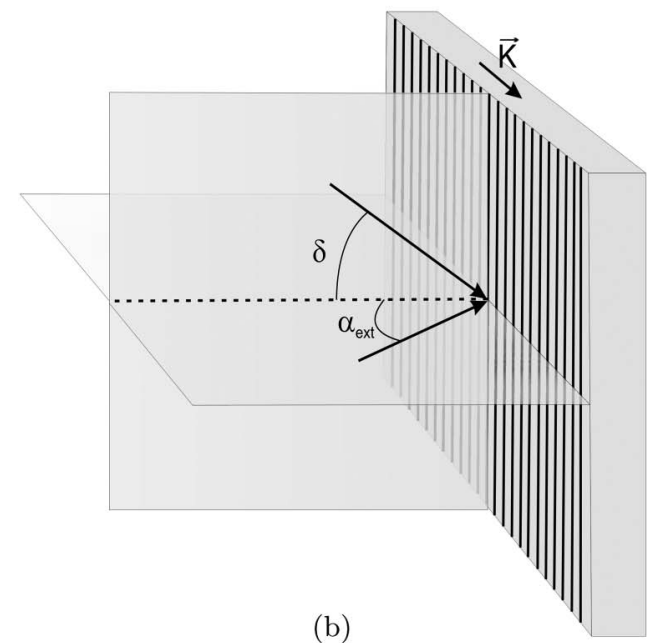

Fig. 6. (a) Hyperspectral generic scheme related to wide-field imager for Earth observation. (b) Grating efficiencies are characterized following the diffraction direction defined by the $\alpha_{\text {ext }}$ angle and the geometric field angle $\delta_{\text {ext }} . \alpha_{\text {ext }}$ and $\delta_{\text {ext }}$ are considered outside the holographic material.

implies that such components could be used in wide-field spectral imagery.

\section{Effect of Gamma Irradiation}

In this work, we have tested the effect of radiation on the VPH grating and specifically on the diffraction efficiency. The high-energy radiation environment in space varies considerably and depends on the period of the launch and the orbital position of the spacecraft. Table $\underline{2}$ lists typical annual radiation doses received by a spacecraft placed in Low Earth Orbit (LEO) and in Geostationary Earth Orbit (GEO). The dose is given in Si-equivalent/year, with $4 \mathrm{~mm}$ Al-equivalent shielding.

VPH grating samples were irradiated by intensity steps from 0 to $120 \mathrm{krad}$. Between each irradiation, the optical transmission spectrum was measured. The value of the diffraction efficiency was then extracted for both polarizations.

The source used is Co-60, which emits $\gamma$-photons with an energy between 1.17 and $1.33 \mathrm{MeV}$. It is known that ionization due to gamma rays provides a useful simulation for penetrating electrons and protons in the space radiation spectrum [29]. For 


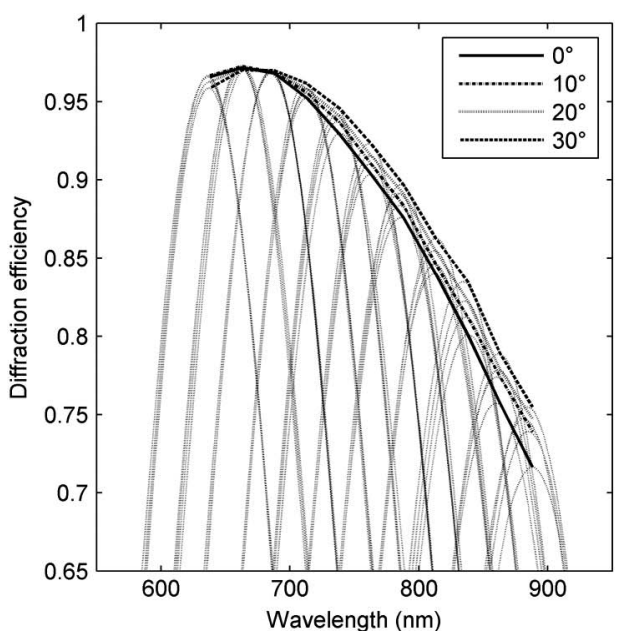

(a) DCG - TM

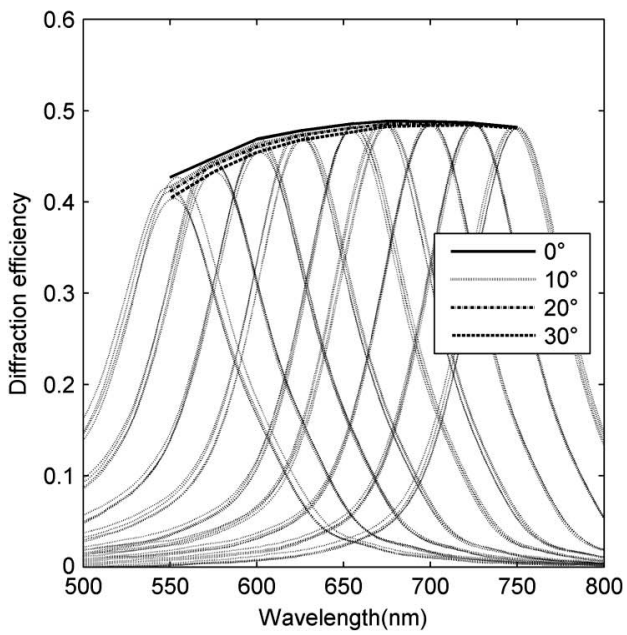

(b) PTR -TE

Fig. 7. Evolution of the diffraction efficiency with the field incidence $\delta$. The range scanned goes from $0^{\circ}$ to $30^{\circ}$. On each graph, the "super blazed" behaviors are represented. Both samples are analyzed in such configurations.

practical purposes, a dose is expressed in $\operatorname{rad}(\mathrm{Si})$ (silicon-equivalent).

Radiation can cause the formation of color centers in optical materials as well as changes in the refractive index and mechanical properties. Changes in the refractive index will produce changes in the focus of optical systems. In the case of VPH gratings, radiations could then change the contrast of the refractive index modulation $\Delta n$ recorded into the sensitive material.

Short wavelength electromagnetic radiation traveling through glass interacts with electrons and

Table 2. Typical Annual Doses Received by Low Earth Orbit and Geostationary Earth Orbit Satellites

\begin{tabular}{cc}
\hline Orbit & Dose $(\mathrm{krad} /$ year $)$ \\
\hline LEO & $1-2$ \\
GEO & $15-20$ \\
\hline
\end{tabular}

atomic nuclei through their electric fields. The most important potential loss mechanisms are the photoelectric effect, Compton scattering, and pair formation. Interaction of the high-energy radiation with optical materials can cause various types of radiation damage. The basic damage mechanisms are the radiolysis by ionization, displacement damage, and electron rearrangement. Ionizing radiation can then produce free holes and electrons in glass can become trapped; thus, forming defect centers. These defect centers cause an increase in the optical absorption in the visible portion of the spectrum leading to a darkening of the glass. For unstabilized optical glasses, total doses of as low as $1 \mathrm{krad}$ of gamma radiation $(1.25 \mathrm{MeV})$ can result in visually detectable coloration. After exposure to hundreds of krad, the transmittance is degraded to the point where the glass may no longer perform adequately in an optical system.

PTR and DCG gratings were submitted to different $\gamma$ doses. For each dose, the diffraction efficiency was evaluated. The evolution of transmittance in the zeroth order is presented in Fig. 8. Each curve is characterized by different $\gamma$ irradiation doses. The dashed curves are the transmittance of the grating stack (cover+gelatin+substrate) at a place where no grating was recorded. In that way, it was possible to retrieve directly the effect of the radiation on the ageing of the material involved in the grating component. In Fig. 9, the results of the diffraction efficiency with the dependence of the gamma dose are presented. On DCG grating, gamma radiation has no significant impact on DCG diffraction efficiencies (TE and TM); whereas, a decrease is observed for PTR. Because of its larger thickness, PTR is more sensitive to gamma radiation.

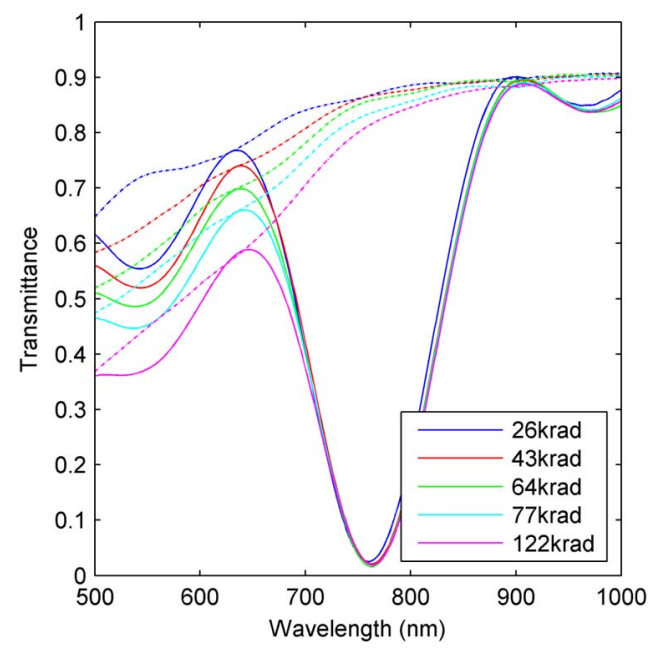

Fig. 8. Transmittance in the zeroth order of TE-diffraction (solid curve) of the DCG grating. Dashed lines correspond to the transmittance of the substrate and cover irradiated under the same condition of $\gamma$-irradiation. From those curves, diffraction efficiency is extracted. The evolution of the diffraction efficiency is then presented in Fig. $\underline{9}$. 


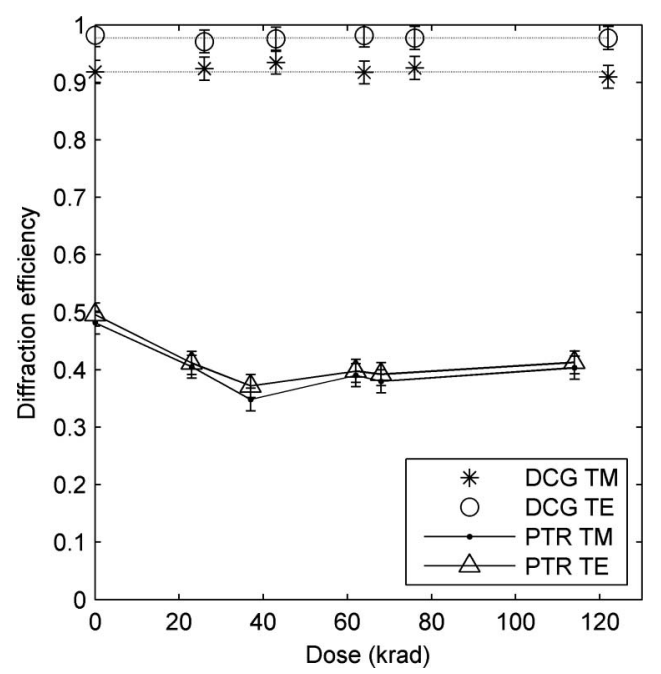

Fig. 9. Evolution with the gamma irradiation dose of the diffraction efficiency at the wavelength design. PTR and DCG diffraction efficiency are presented for both polarizations.

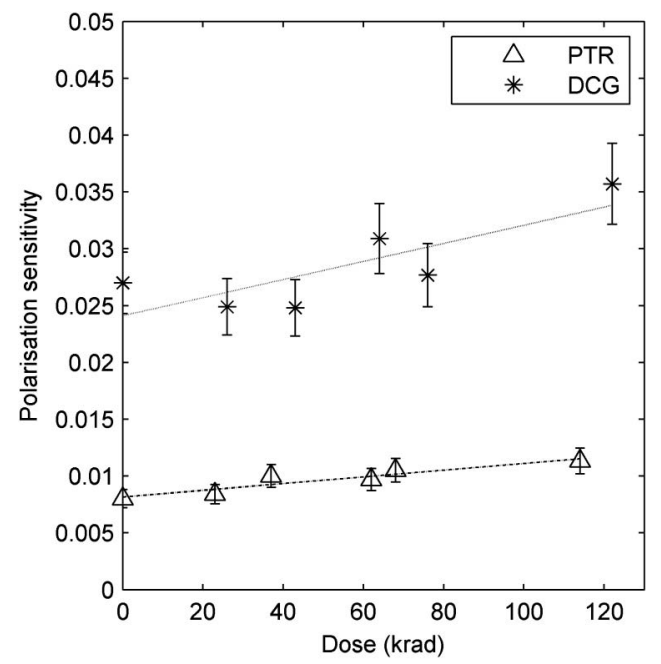

Fig. 10. Polarization sensitivity evolution at the wavelength design as a function of the gamma dose.

The evolution of the polarization sensitivity has also been analyzed. As expected, gamma rays also have an impact on the polarization sensitivity. Figure 10 exhibits the dependency of the polarization sensitivity with respect to the dose. Even if a modification is observable, the effect is very small.

\section{Conclusion}

In this paper, we have attempted to demonstrate the usefulness of VPH gratings for space applications generally and the Earth Observation Mission in particular. The work described in this paper can be extended to other space missions. The objectives were to evaluate the capacity of the VPH gratings to maintain their diffractive properties under space conditions. We focused our objectives on the high-energy radiation environment. As dictated by the ESA requirements, the space radiation environment [29] could be experimentally reproduced for optical components by means of gamma-irradiation of around 1.20 MeV. We then compared two types of Volume Bragg Gratings, which each have useful advantages.

PTR presents interesting behavior concerning polarization sensitivity that is less than half of that for DCG-fabricated gratings. However, for both gratings, PTR and DCG, the polarization sensitivity property is largely better than for SR gratings. Again, DCG has more interesting properties concerning the radiation resistance than PTR. Furthermore, bandwidths achievable with DCG grating are significantly larger than those achievable with the PTR. In conclusion, in the frame of hyperspectral/multispectral missions, DCG-gratings are more suitable because of bandwidth, diffraction efficiency, and radiation survivability. The drawbacks of PTR lie in its limited bandwidth spectral component. However they can be used as very narrow bandwidth filter.

The authors would like to thank the European Space Agency for the financing of this study under contract N 22616/09/NL/RA.

\section{References}

1. P. Harihaman, Basics of Holography (Cambridge University, 2002).

2. R. J. Collier, C. B. Burckhardt, and L. H. Lin, Optical Holography (Academic, 1971).

3. S. C. Barden, J. A. Arns, and W. S. Colburn, "Volume-phase holographic gratings and their potential for astronomical applications," Proc. SPIE 3355, 866-876 (1998).

4. P. Blanche, P. Gailly, S. Habraken, P. Lemaire, and C. Jamar, "Volume phase holographic gratings: large size and high diffraction efficiency," Opt. Eng. 43, 2603-2612 (2004).

5. C. Kitchin, Astrophysical Techniques (IOP, 1991).

6. D. Schroeder, Astronomical Optics (Academic, 2000).

7. O. Efimov, L. Glebov, and V. Smirnov, "High-frequency Bragg gratings in photothermorefractive glass," Opt. Lett. 25, 1693-1695 (2000).

8. L. Glebov, "Volume hologram recording in inorganic glasses," Glass Sci. Technol. 75, 73-90 (2002).

9. H. Kogelnik, "Coupled-wave theory of thick hologram gratings,” AT\&T Tech. J. 48, 2909-2947 (1969).

10. M. G. Moharam and T. K. Gaylord, "Rigorous coupled-wave analysis of planar-grating diffraction," J. Opt. Soc. Am. 71, 811-818 (1981).

11. M. G. Moharam and T. K. Gaylord, "Three-dimensional vector coupled-wave analysis of planar-grating diffraction," J. Opt. Soc. Am. 73, 1105-1112 (1983).

12. A. Bianco, G. Pariani, A. Zanutta, and C. Bertarelli, "Practical considerations in the case of astronomical instrumentation," Proc. SPIE 8450, 84502W(2012).

13. H. I. Bjelkhagen, Silver-Halide Recording Materials: For Holography and Their Processing (Springer-Verlag, 1995).

14. T. Shankoff, "Phase holograms in dichromated gelatin," Appl. Opt. 7, 2101-2105 (1968).

15. J. Chang and C. D. Leonard, "Dichromated gelatin for the fabrication of holographic optical elements," Appl. Opt. 18, 2407-2417 (1979).

16. S. Martin, C. Feely, and V. Toal, "Holographic recording characteristics of an acrylamide-based photopolymer," Appl. Opt. 36, 5757-5768 (1997).

17. J. Lawrence, F. O'Neill, and J. Sheridan, "Photopolymer holographic recording material," Optik 112, 449-463 (2001).

18. B. L. Booth, "Photopolymer material for holography," Appl. Opt. 14, 593-601 (1975).

19. H. Bouas-Laurent and H. Durr, "Organic photochromism," Pure Appl. Chem. 73, 639-665 (2001). 
20. J. Crano, T. Flood, D. Knowles, A. Kumar, and B. V. Gemert, "Photochromic compounds: chemistry and application in ophthalmic lenses," Pure Appl. Chem. 68, 1395-1398 (1996).

21. J. Loicq, M. Georges, and L. Venancio, "Investigation on the high efficiency volume Bragg gratings performances for spectrometry in space environment: preliminary results," Proc. SPIE 8442, 84424Z (2012).

22. V. Natraj, R. Spurr, H. Boesch, Y. Jiang, and Y. Yung, "Evaluation of errors from neglecting polarization in the forward modeling of $\mathrm{O}_{2} \mathrm{~A}$ band measurements from space, with relevance to $\mathrm{CO}_{2}$ column retrieval from polarizationsensitive instruments," J. Quant. Spectrosc. Radiat. Transfer 103, 245-259 (2007).

23. E. Boesche, P. Stammes, and R. Bennartz, "Aerosol influence on polarization and intensity in near-infrared $\mathrm{O}_{2}$ and $\mathrm{CO}_{2}$ absorption bands observed from space," J. Quant. Spectrosc. Radiat. Transfer 110, 223-239 (2009).

24. C. Plamer and E. Loewen, Diffraction Grating Handbook (Newport Corporation, 2005)

25. E. Loewen, M. Neviere, and D. Maystre, "Efficiency optimization of rectangular groove gratings for use in the visible and IR regions," Appl. Opt. 18, 2262-2266 (1979).

26. M. G. Moharam and T. K. Gaylord, "Diffraction analysis of dielectric surface-relief gratings," J. Opt. Soc. Am. 72, 1385-1392 (1982).

27. K. Yokomori, "Dielectric surface-relief gratings with high diffraction efficiency," Appl. Opt. 23, 2303-2310 (1984).

28. C. Donlon, "Sentinel-3 Mission Requirements Trace-ability Document," Technical report EOP-SM/2184/CD-cd (ESA, 2011).

29. "The Radiation Design Handbook," ESA PSS-01-609 (ESA, 1993) 